RESEARCH NOTE

\section{Echinopardalis sp.}

(Acanthocephala, Oligacanthorhynchidae) Eggs in Felid Coprolites Dated From 9,000 Years Before Present, Found in the Brazilian Northeast

\section{Dely Noronha, Luiz Fernando Ferreira*, Adriana Rangel*/+, Adauto Araujo*, Delir Corrêa Gomes ${ }^{+}$}

Laboratório de Helmintos Parasitos de Vertebrados, Departamento de Helmintologia, Instituto Oswaldo Cruz *Departamento de Ciências Biológicas, Escola Nacional de Saúde Pública,

Av. Brasil 4365, 21045-900 Rio de Janeiro, RJ, Brasil

Key words: Echinopardalis sp. - coprolites

Acanthocephalan eggs were found in felid coprolites dated of 9,000 years BP, at the archeological site of São Raimundo Nonato, State of Piaui, in the northeast of Brazil ( $8^{\circ} 51^{\prime} \mathrm{S}$, $\left.42^{\circ} 33^{\prime} \mathrm{W}\right)$. They were identified as Echinopardalis sp., after comparison with the eggs of Oncicola Travassos, 1916 and Neoncicola Schmidt, 1972.

Animal coprolites revealed parasite infections dated of 32,000 years BP showing ancient parasite/host relationships, and supporting theories about climatic changes that occurred 10,000 years ago (LF Ferreira et al. 1991 J Parasitol 77: 491493).

The classification adopted herein is the one of BB Nickol and TT Dunagan (1989 Proc Helminthol Soc Wash 56: 8-13). It is worthwhile to emphasize that the first referred author (Nickol) a co-author of the paper by JFR Amato et al. (1979 Proc Helminthol Soc Wash 46: 279-281) was responsible for the reconsideration of the status of the genus Echinopardalis (1989), when in a checklist, includes the herein reported species in the genus Echinopardalis.

One hundred and twenty one animal coprolites dated of 9,000 years BP by the radiocarbon method were collected and examined for para-

Support by CNPq.

${ }^{+} \mathrm{CNPq}$ research fellows

Received 18 June 1993

Accepted 30 November 1993 sites. Identification of zoological origin was obtained by comparisons with recent desiccated feces of present-day animals of the region according to Chame et al. (1989 Paleopathol News 68: 9-11, 1991, 76: 7-9).

Standard techniques for rehydratation and parasitological examination were used, after $\mathrm{O}$ Callen and WM Cameron (1960 New Sci 8: 3540), and K Reinhard et al. (1988 Homo 37: 217 239). Twenty-three samples were identified as felid coprolites.

Additional examinations of acanthocephalan species, namely Oncicola oncicola (Ihering, 1902) Schmidt, 1972, Neoncicola potosi Machado Filho, 1950, Echinopardalis lamasi Freitas \& Costa, 1964 and E. pardalis (Westrumb, 1821) Travassos, 1918, deposited as whole mounts in the Helminthological Collection of the Instituto Oswaldo Cruz (CHIOC), no. 28.983, 17.829b, $31.798 \mathrm{~b}$ and $33.045 \mathrm{a}-\mathrm{e}$, respectively, were made. Preserved material of eggs in coprolites was also prepared as whole mounts and deposited in the CHIOC under no. 33.058a-f.

Measurements are in micrometers unless otherwise indicated. Means are within parentheses. Drawings were made with a drawing tube, connected to a Leitz light microscope.

Two out of the 23 coprolites identified as felid fecal material (Fig. 1) by morphometric parameters and contents, such as fragments of bones and feathers, showed parasite eggs identified as eggs of the acanthocephalan Echinopardalis $\mathrm{sp}$.

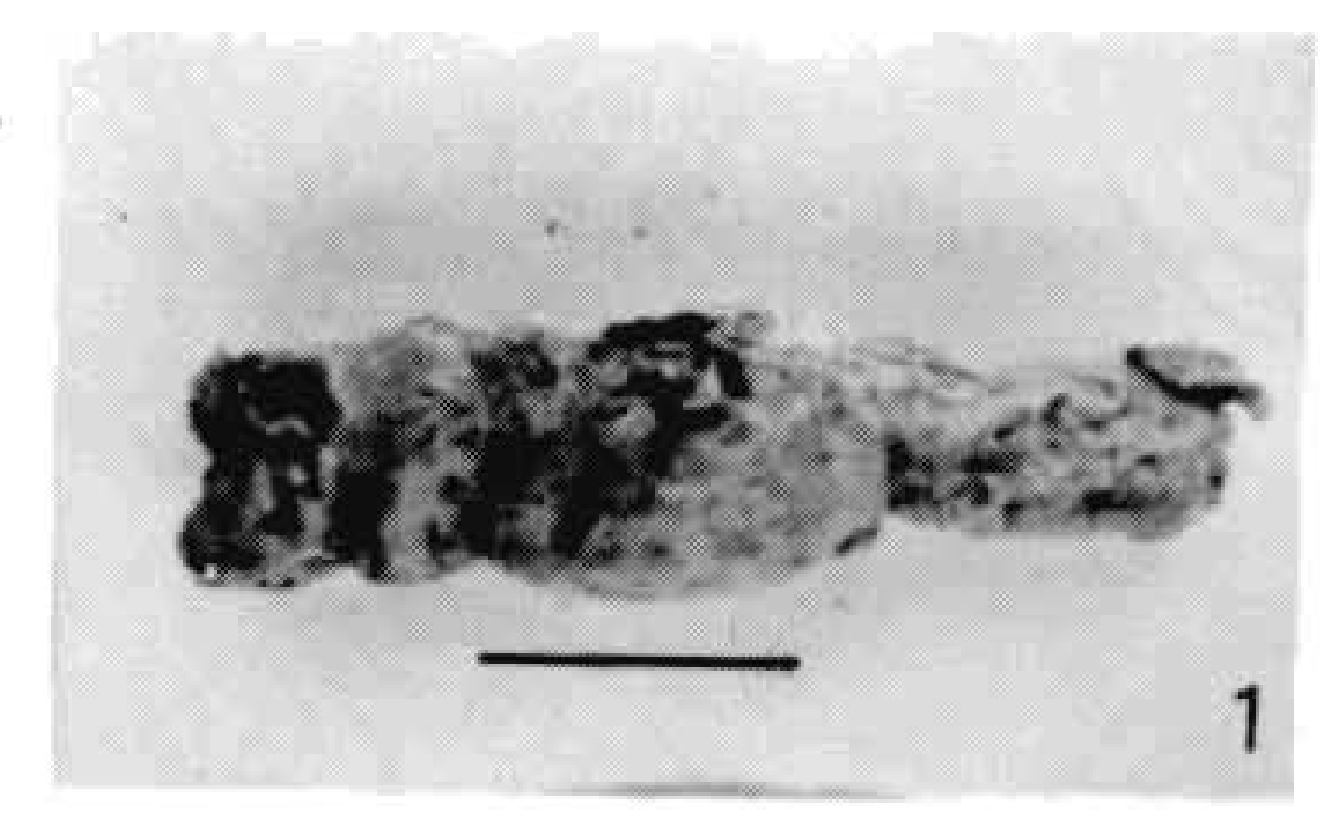

Fig. 1: felid fecal material (coprolite). $\mathrm{Bar}=2 \mathrm{~cm}$.

In order to reach the generic identification for the eggs found in the coprolites, the shape of the eggs and pattern of host distribution near the collection site have been considered.

The eggs were oval, with concentric membranes and without external shell, 56-66 (60) long by 43-49 (47) wide (Figs 2, 5). The measurements were based on eight eggs.

Based on comparisons made with the eggs of Oncicola (Fig. 3), Neoncicola (Fig. 4) and Echinopardalis (Fig. 6), the eggs found in the 


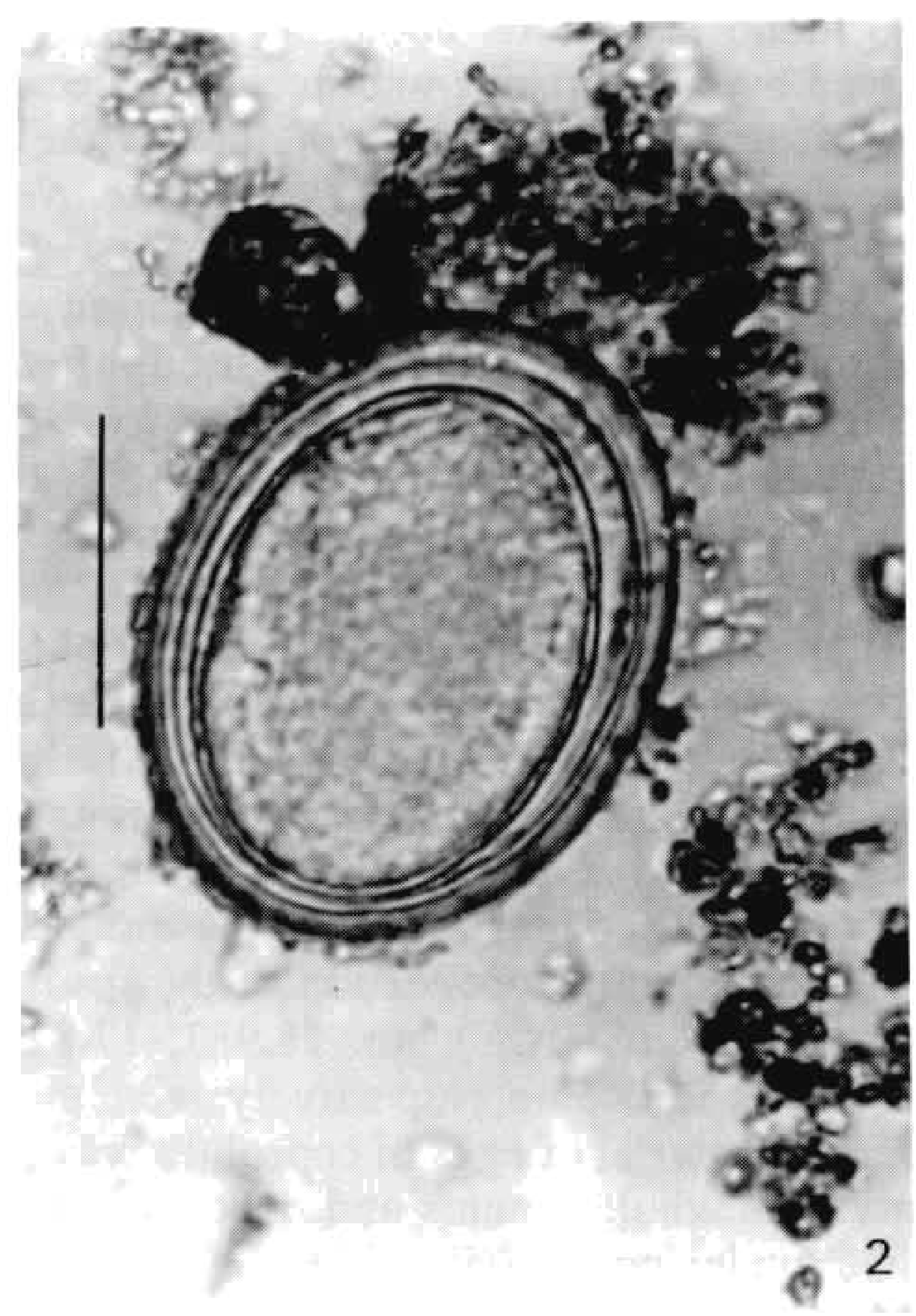

Fig. 2: Echinopardalis sp - egg. Bar $=0.027 \mathrm{~mm}$.
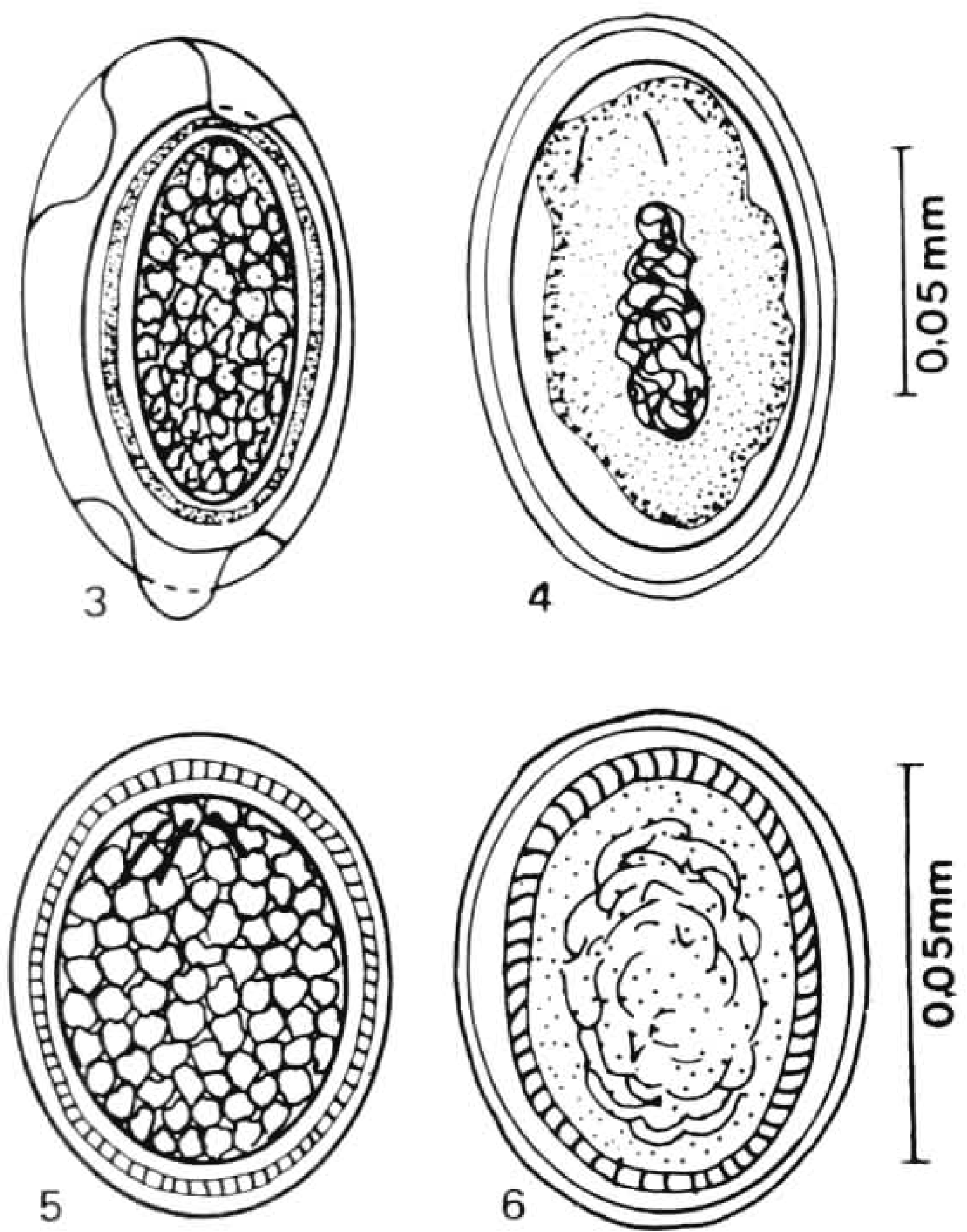

Fig. 3: Oncicola oncicola - egg. Fig. 4: Neoncicola potosi - egg. Fig. 5: Echinopardalis sp. - egg. Fig. 6: E. pardalis - egg (after Travassos, 1917). Bar $=0.05 \mathrm{~mm}$. coprolites were identified as those of the latter genus. Eggs of Neoncicola present a sculptured outer membrane, while those of Oncicola are broadly ellypsoidal, without a compact external membrane.

The genus Echinopardalis was originally proposed by L Travassos (1917 Brazil Medico 31: 121-122) in a brief description as Pardalis, when a checklist of Giganthorhynchidae acanthocephalans deposited in the "Museu Paulista" in São Paulo, State of São Paulo, Brazil, was published.

In the same year, L Travassos (1917 Mem Inst Oswaldo Cruz 9: 5-62), revising the Brazilian Acanthocephala, completed the description with figures and host lists.

Later, L Travassos (1918 Communication in Seção de Sciencias Biologicas: 235) renamed the genus as Echinopardalis, since Pardalis was preocupied and the first published full paper to mention the new arrangement was that of $\mathrm{L}$ Travassos (1920 Rev Vet Zootech 10: 3-23).

All the bibliographical data related to the paper in which appeared the designation of the genus as Echinopardalis (1918), have been erroneous since then.

The generic identification reported here for the archeological material is also based on the fact that four species of the genus Echinopardalis have been reported from Brazil, parasitizing felid hosts.

The morphology and size of the coprolites point to the small wild cats and since Felis geoffroyi has not been recorded in the region, $F$. tigrina and $F$. pardalis are the likely hosts. Other small felids in the region are $F$. wiedi Schinz, 1821 and $F$. yaguaroundi Geoffroy, 1803.

Acknowledgement: to Mara Lucia de Souza Lemos, IOC research fellow, from the Setor de Programação Visual (SICT/FIOCRUZ) for graphic revision of figures. 